\title{
UM ENSAIO SOBRE O EFEITO DISPOSIÇÃO NA REALIZAÇÃO DE GANHOS E PERDAS EM INVESTIMENTOS
}

Robson Braga'

Luiz Paulo Fávero²

Resumo: O efeito disposição está relacionado à tendência com que as pessoas realizam mais rapidamente a venda de ativos com ganhos em relação àqueles com perdas. Desde os seminais $\mathrm{e}$ precursores estudos sobre o tema, diversas pesquisas foram realizadas sobre o assunto e demonstram que o efeito disposição parece estar longe de estar plenamente explicado. Em virtude dessa aparente falta de consenso sobre o assunto, este ensaio apresenta uma análise da produção científica sobre o efeito disposição no período compreendido entre 2010 e 2014, complementando estudo anterior que apresentou levantamento sobre as pesquisas sobre o efeito disposição no período entre 1985 e 2009. Por meio de uma metodologia empírico-analítica, foi realizada análise de trinta e seis trabalhos identificados em periódicos nacionais e internacionais sobre o efeito disposição, cujos resultados são apresentados e discutidos por meio de tabelas, gráficos e redes científicas. Os achados obtidos mostram que houve mudanças nos procedimentos metodológicos dos estudos mais recentes, com utilização de novos modelos estatísticos para explicar o efeito disposição e suas variantes, além da observação de outros fenômenos associados ao efeito disposição no contexto do processo de tomada de decisão em investimentos.

Palavras-chave: Efeito Disposição. Teoria do Prospecto. Realização de Ganhos e Perdas. Decisão de Investimento. 


\title{
AN ESSAY ON THE DISPOSITION EFFECT IN THE REALIZATION OF GAINS AND LOSSES ON INVESTMENTS
}

\begin{abstract}
The disposition effect is related to the tendency that people make faster gains than losses. Since seminal and precursor published studies in the field, lots of researches were performed on the subject and show that the disposition effect seems to be far from being fully explained. Because of this apparent lack of consensus on the subject, this essay presents an analysis of scientific literature on the disposition effect in the period between 2010 and 2014, continuing a previous research that presented a survey on the disposition effect between 1985 and 2009. Through an empirical-analytical methodology, we carried out an analysis of thirty-six works on disposition effect published in national and international journals, and the results are presented and discussed through tables, graphs and scientific networks. The findings show that, recently, there is a change in methodological procedures to investigate the theme, with the introduction of new statistical models and new types of observation of the phenomenon, what allows one to better understand the decision making process on investments.
\end{abstract}

Keywords: Disposition Effect. Prospect Theory. Realization of Gains and Losses. Investment Decision. 


\section{INTRODUÇÃO}

$\mathrm{D}$ esde que os estudos sobre finanças comportamentais começaram a chamar a atenção da comunidade cientifica, vários temas têm sido objeto de investigação em diversas áreas. Dentre esses temas, boa parte das investigações deriva dos fundamentos da teoria do prospecto (Kahneman \& Tversky, 1979) e busca compreender o comportamento do investidor em contextos que envolvem ganhos e perdas. É o caso do efeito disposição, que está associado com a tendência que as pessoas têm de realizar cedo demais a venda de ativos com ganhos e manter aqueles com perdas (Shefrin \& Statman, 1985).

A identificação desse fenômeno ocorreu a partir da observação de que os investidores mantêm em suas carteiras ações vencedoras ou lucrativas por menos tempo do que as perdedoras (Odean, 1998). Esse comportamento contraria os fundamentos da racionalidade, uma vez que não contribui para a maximização do retorno da carteira de investidores, pelo contrário, tende a elevar as perdas (Hens e Vlcer, 2011).

Conforme apontam Talpsepp, Vleck e Wang (2014), diferentes explanações têm buscado compreender esse frequente padrão de comportamento que atinge os investidores. Existem associações diretas com a aversão ao risco, oriunda da teoria do prospecto (Kahneman \&Tversky, 1979), a um viés cognitivo denominado contabilidade mental (Thaler, 1985) e a aspectos comportamentais diversos, dentre eles a busca por "vencer" o mercado, operando de maneira mais ativa (Odean, 1998).

A maior parte dos estudos tem assumido o efeito disposição como sendo objetivamente explicado pela atitude assimétrica ao risco, como proposto pela teoria do prospecto (Talpsepp, Vleck \& Wang, 2014). Por esse entendimento, as pessoas são mais avessas ao risco quando estão ganhando e buscam mais o risco quando estão perdendo (Kahneman \& Tversky, 1979). Os fundamentos da teoria do prospecto, contudo, não têm sido suficientes para explicar o efeito disposição (Henderson, 2012).

A crença na reversão dos preços à média tem sido outra linha explanatória (Hens \& Vlcek, 2006). Acreditar na tendência de que os preços se reverteriam à média leva à crença de que as ações perdedoras de hoje serão as vencedoras de amanhã. Com isso, os investidores acreditariam que não valeria a pena vender ações que podem render bons lucros no futuro (Costa Junior, Mineto \& Silva, 2008). Esse entendimento, aliado a outros fatores, pode comprometer a avaliação do comportamento do investidor como sendo típico do efeito disposição (Ben-David \& Hirshleifer, 2012).

A relevância do efeito disposição no âmbito dos estudos sobre comportamento do investidor, o surgimento de outros aspectos relacionados com o processo decisório no mercado de ações, que impactam a decisão de venda de ações ganhadoras e perdedoras, e o fato de os estudos sofre efeito disposição apresentarem resultados controversos, como destaca Henderson (2012), torna relevante sua investigação. Por isso, este estudo busca responder à seguinte questão de pesquisa: quais as explicações encontradas na literatura sobre o efeito disposição para a tendência do investidor em vender ações vencedoras e manter ações perdedoras?

Uma vez que não existe consenso entre os estudiosos sobre o que explica o efeito disposição (Hens \& Vleck, 2006), avaliar em que direção os achados mais recentes apontam, é relevante para compreender melhor esse que é um fenômeno recorrente no âmbito das decisões de investimento. O objetivo 
desse estudo é relacionar as evidências dos estudos sobre o efeito disposição e o comportamento dos investidores no mercado de ações no período de 2010 a 2015. Lucchesi (2010) realizou levantamento dessa natureza sobre o efeito disposição entre o período de 1985 a 2009, sendo esse estudo complementar ao do pesquisador.

Para tanto, é feita análise dos achados de artigos publicados nos periódicos de finanças e contabilidade internacionais, que realizaram pesquisas empíricas sobre o efeito disposição. Procedimento metodológico semelhante foi originalmente utilizado por D'Souza e Lima (2014), que buscaram identificar a produção científica a respeito da tríade conhecida como Dark Triad, que afeta o comportamento decisório de gestores. A análise desses achados é apresentada por meio de tabelas com os resultados dos estudos e rede científica que relaciona os pesquisadores, a metodologia de suas pesquisas e as evidências empíricas de seus estudos.

\section{REFERENCIAL TEÓRICO}

\subsection{TEORIA DO PROSPECTO}

A teoria do prospecto derivou de estudos realizados por Kahneman e Tversky (1979) envolvendo escolhas em condições de risco. Por meio de diversos experimentos, nos quais os participantes tinham que fazer escolhas entre ganhos e perdas mais ou menos certos, os pesquisadores perceberam haver um comportamento diverso das pessoas quando estão diante de opções de mais risco de ganhos ou de perdas.

Henderson (2012) destaca que na teoria do prospecto o conceito de utilidade é definido sobre ganhos e perdas a partir de um ponto de referência ao invés do nível de riqueza final, conforme ideia proposta por Markowitz (1952). Com isso, a função utilidade derivada da teoria do prospecto exibe concavidade para o domínio dos ganhos e convexidade no domínio das perdas (Kahneman, 2012).

Segundo Ferreira (2008), a teoria do prospecto mostra que as pessoas no contexto das perdas buscam mais risco, enquanto que em momentos de ganhos elas preferem obter um lucro menor, mas certo, a buscar arriscar ganhar mais, de modo incerto. A isso, Kahneman e Tversky (1979) denominam "efeito certeza" (certainty effect). A teoria do prospecto aponta ainda o efeito isolamento (isolation effect), que faz com que as pessoas façam escolhas inconsistentes quando as mesmas opções são apresentadas de maneira diferente.

Kahneman e Tversky (1979) indicam que as pessoas têm comportamento adverso frente a escolhas que apresentam maior ou menor chance de ganho ou perda. Para Ferreira (2008) "em contextos de ganho, o efeito certeza contribui para uma preferência com aversão a risco em relação a um ganho certo, quando comparado a um ganho maior que seja meramente provável".

A teoria do prospecto identifica três características cognitivas que a sustentam: a avaliação é relativa a um ponto de referência neutro, que é tipicamente considerado como status quo ou o resultado que se espera em uma decisão; o princípio de sensibilidade subjetiva decrescente entre valores; e o princípio da aversão à perda, um das mais relevantes no contexto dos investimentos (Thaler, 2008).

A aversão à perda e o ponto de referência do valor investido fazem com que muitos investidores fiquem relutantes em realizar perdas em suas aplicações (Shefrin \& Statman, 1985). Esse fenômeno ficou conhecido como efeito disposição (disposition effect) e representa a observação de que os investidores desproporcionalmente vendem ativos vencedores e mantêm aqueles com perdas (Henderson, 2012). 


\subsection{EFEITO DISPOSIÇÃO}

Terrance Odean desenvolveu diversos estudos sobre comportamento do investidor no mercado financeiro, alguns deles ao lado de seu colega Brad Barber. Em seus estudos, cabe destacar aqueles referentes a decisões de investimento no mercado financeiro, tais como aqueles com objetivo de avaliar o comportamento dos investidores frente a decisões de compras e vendas de ações (Odean, 1998), excesso de operações no mercado financeiro (Barber e Odean, 2000) e diferença de gênero em decisões de investimentos (Barber e Odean, 2001).

Sobre o efeito disposição, o estudo de Odean (1998) é considerado uma referência das mais relevantes (Lucchesi, 2010), uma vez que o pesquisador teve a oportunidade de avaliar o resultado de mais de 160.000 operações de milhares de contas de investidores de uma corretora de valores. $O$ teste realizado pelo autor, denominado de teste de diferença de proporção, se baseou nos seguintes cálculos:

\begin{tabular}{|c|c|}
\hline Proportion of Gains Realized (PGR) $=$ & Realized Gains \\
\hline & Realized Gains + Paper Gains \\
\hline Proportion of Losses Realized (PLR) $=$ & Realized Losses \\
\hline & Realized Losses + Paper Losses \\
\hline
\end{tabular}

Uma grande diferença na proporção de ganhos realizados (PGR) e a proporção de perdas realizadas (PLR) indica que investidores são mais dispostos a realizar ganhos do que perdas, de acordo com a prevalência da maior proporção (Odean, 1998). Esse método de diferença de proporção é um dos mais utilizados em estudos sobre o efeito disposição, como demonstra levantamento feito por Lucchesi (2010).

A partir dessa proposição, os resultados apurados por Odean (1998) apontaram para a existência do efeito disposição, sendo os investidores propensos a vender ações vencedoras (winners) ou com ganhos (gains) e manter as perdedoras (losers) ou com perdas (losses). A exceção foi verificada no mês de dezembro, em que ocorre a venda de ações perdedoras, em virtude do ajuste de posição para fins de benefício tributário (tax loss selling).

O efeito disposição tem suas bases nos fundamentos da teoria do prospecto, considerando o padrão de escolha apresentado pelos investidores quando estão diante de decisões que envolvem ganhos e perdas (Shefrin \& Statman, 1985; Odean, 1998). Segundo Thaler (2008), que apresentou demonstrações disso por meio da identificação da aversão à perda em apostas, as pessoas odeiam perder.

Para o pesquisador, a aversão à perda ajuda a produzir inércia, fazendo com que as pessoas desejem fortemente que as coisas permaneçam como estão. Esse desejo gera um comportamento adverso, chamado viés do status quo. Samuelson e Zeckhauser (1988) destacam que em situações reais, frente a problemas que precisam de soluções efetivas, as pessoas consideram a opção do status quo, considerado simplesmente "não fazer nada" como opção viável e muitas vezes preferível.

No contexto do efeito disposição, o viés do status quo aparece no momento em que as pessoas decidem não vender (ou seja, "não fazer nada") suas ações perdedoras, acreditando que em algum momento no futuro os preços irão retornar ao ponto de referência que eles guardam como resultado de uma contabilidade mental (Thaler \& Sunstein, 2008).

Sobre a contabilidade mental Thaler e Sunstein (2008) asseveram se tratar de um sistema de controle interno utilizado pelos indivíduos para avaliar, regular e realizar seus processos pessoais. Os tomadores de decisões se valeriam dela para fins de configuração de seus pontos de referência para os valores 
que determinam ganhos e perdas (Grinblatt \& Han, 2004). Relacionando os fundamentos da teoria do prospecto sobre ganhos e perdas com a contabilidade mental, os pesquisadores destacam que os tomadores de decisões muitas vezes deixam de considerar as interações existentes entre as diversas apostas ou investimentos, podendo elevar as chances de ocorrência do efeito disposição.

Emana da contabilidade mental um viés cognitivo, denominado efeito dotação (endowment effect), que diz respeito à tendência que as pessoas têm de supervalorizar aquilo que elas têm, especialmente quando a posse se refere a bens que se tem com o objetivo de uso e não de troca, conforme experimentos realizados por Kahneman, Knetsch e Thaler (1990). Os pesquisadores realizaram estudo envolvendo trocas e notaram que quando os produtos em posse eram vistos como um meio para a obtenção de algo mais valioso, a abertura à negociação era mais franca do que quando a posse era de produtos com objetivo de uso final.

O efeito disposição apresenta interfaces com vários vieses cognitivos e comportamentos adversos assumidos pelos tomadores de decisão em investimentos. Isso faz com que os pesquisadores apresentem discussões divergentes para explicar os achados empíricos de seus estudos sobre o efeito disposição, tais como rebalanceamento de portfólio, crença na reversão à média, operação realizada com base em informação privada, operações motivadas por questões tributárias ou por custos de transação (Henderson, 2012).

Em função dessas divergências, Henderson (2012) destaca ainda que muitos autores têm encontrado dificuldade de predizer se os achados realmente correspondem ao efeito disposição. Por outro lado, destaca o pesquisador, existem aqueles que encontram forte efeito disposição, com identificação de investidores que vendem ações com ganhos e relutam em voluntariamente vender as perdedoras (Henderson, 2012).

Talpsepp, Vleck eWang (2014) destacam que a identificação do efeito disposição tem sido observada em diversos mercados, citando os trabalhos de Odean (1998) nos Estados Unidos, Feng e Seasholes (2005) na China e Grinblatt e Keloharju (2001) na Finlândia. No contexto brasileiro, destacam-se os trabalhos de Costa Junior, Mineto e Silva (2008), que identificaram o efeito disposição por meio de estratégias metodológicas experimentais. Os pesquisadores consideram a existência de ilusão cognitiva, viés oriundo da aversão à perda e expectativa de reversão à média como potenciais fatores explicativos do efeito disposição.

Lucchesi (2010), além dos seus próprios achados, fez um levantamento sobre os estudos empíricos realizados sobre o efeito disposição desde a pesquisa seminal de Shefrin e Statman (1985) até Barberis e Xiong (2009). Ou seja, o pesquisador realizou um levantamento dos trabalhos mais relevantes sobre o efeito disposição de 1985 até 2009, totalizando vinte e três trabalhos, com vistas a "harmonizar os vários pontos de vista, a fim de oferecer uma visão panorâmica do estado da questão" (Lucchesi, 2010).

Em seu estudo, Lucchesi (2010) analisou vinte e três trabalhos, produzidos no Brasil e no mundo no período compreendido entre 1985 e 2009. O pesquisador discute os achados de cada um dos estudos referenciados, especialmente no que se refere aos critérios metodológicos, apresentando um quadro sintético fundamentando as bases de dados utilizadas. O Quadro 1 apresenta uma síntese das evidências empíricas dos referidos trabalhos a partir dos resultados obtidos pelos pesquisadores que estudaram o efeito disposição no período de 1985 a 2009. 
Quadro 1: Síntese das evidências dos estudos sobre efeito disposição de 1985-2009

\begin{tabular}{|c|c|}
\hline Estudo & Evidências empíricas \\
\hline Shefrin e Statman (1985) & $\begin{array}{l}\text { Em três escalas de tempo distintas, os investidores individuais e os gestores de fundos mútuos } \\
\text { manifestaram o efeito disposição, pelas vendas em maior proporção de ativos com ganhos em } \\
\text { relação àqueles com perdas. }\end{array}$ \\
\hline Lakonishok e Smidt (1986) & $\begin{array}{l}\text { A partir de uma janela temporal de até quase três anos, os pesquisadores identificaram tendência ao } \\
\text { efeito disposição, evidenciando, porém, que a busca por compensações tributárias (tax-loss-selling) } \\
\text { faz com que a venda de ações perdedoras cresça no fim do ano. }\end{array}$ \\
\hline Ferris, Haugen e Makhija (1988) & $\begin{array}{l}\text { Os pesquisadores relacionaram o efeito disposição com o tax-loss-selling por meio de um estudo } \\
\text { comparativo de negociações históricas com atuais. Identificaram padrões convergentes com o efeito } \\
\text { disposição, mas inconclusivos para o tax-loss-selling. }\end{array}$ \\
\hline Odean (1998) & $\begin{array}{l}\text { A partir de uma ampla base de dados, o pesquisador reforça a hipótese das negociações fundadas } \\
\text { no tax-loss-selling, além de identificar um comportamento dos investidores de expectativa de } \\
\text { reversão dos preços à média por parte das ações perdedoras. }\end{array}$ \\
\hline Weber e Camerer (1998) & $\begin{array}{l}\text { Por meio de um estudo experimental, tendo como referência duas bases de preços, os } \\
\text { pesquisadores identificaram tendência dos participantes ao efeito disposição, a partir da } \\
\text { comparação das proporções de compras e vendas de ações vencedoras e perdedoras. }\end{array}$ \\
\hline Grinblatt e Keloharju (2000) & $\begin{array}{l}\text { A partir da avaliação das negociações das principais ações do mercado finlandês durante dois anos, } \\
\text { os pesquisadores identificaram uma tendência dos investidores individuais a comprarem mais ações } \\
\text { perdedoras do que vencedoras. Tal comportamento foi inverso entre os investidores institucionais. }\end{array}$ \\
\hline Boebel e Taylor (2000) & $\begin{array}{c}\text { Por meio de uma avaliação do efeito disposição com uso de um indicador estatístico e dos preços } \\
\text { médios de negociação das ações. Os resultados mostraram resultados divergentes para o efeito } \\
\text { disposição quando da avaliação pelo preço médio e pelo indicador técnico chamado índice de força } \\
\text { relativa (IFR). }\end{array}$ \\
\hline Locke e Mann (2000) & $\begin{array}{l}\text { Os pesquisadores avaliaram o comportamento de operadores profissionais de mercados futuros de } \\
\text { commodities e identificaram, dada a manifestação do efeito disposição, havia uma relação negativa } \\
\text { entre a manifestação do efeito disposição e o desempenho dos contratos. }\end{array}$ \\
\hline Ranguelova (2001) & $\begin{array}{l}\text { Por meio de metodologia semelhante à de Odean (1998), o estudo validou o efeito tax-loss-selling } \\
\text { e evidências do efeito disposição. Porém, identificou que o nível de capitalização das empresas } \\
\text { influencia o comportamento do investidor em relação à retenção de ações perdedoras ou } \\
\text { vencedoras. }\end{array}$ \\
\hline Shapira e Venezia (2001) & $\begin{array}{l}\text { Os pesquisadores avaliaram o tempo de permanência de ações vencedoras e perdedoras nas } \\
\text { carteiras de clientes que operam por conta própria e dos que contam com apoio nas decisões de } \\
\text { compra e venda. Perceberam que o efeito disposição é maior entre os clientes independentes. }\end{array}$ \\
\hline Genesove e Mayer (2001) & $\begin{array}{l}\text { Os pesquisadores se valeram dos fundamentos do efeito disposição para avaliar o comportamento } \\
\text { de proprietários de imóveis e notaram que a aversão à perda, oriunda do efeito disposição, elevava } \\
\text { as perdas dos vendedores que exigiam preços muito acima dos praticados pelo mercado. }\end{array}$ \\
\hline Grinblatt e Han (2002) & $\begin{array}{l}\text { Por meio do uso de um indicador de análise técnica que avalia o comportamento e a tendência } \\
\text { dos preços das ações (momentum) os pesquisadores identificaram que a manifestação do efeito } \\
\text { disposição por parte dos investidores afeta os preços das ações, gerando um spread adicional. }\end{array}$ \\
\hline Dhar e Zhu (2002) & $\begin{array}{l}\text { Utilizando uma ampla base de dados, semelhante ao estudo de Odean (1998), os pesquisadores } \\
\text { identificaram que os investidores com maior nível de informação demonstravam um grau de efeito } \\
\text { disposição muito menor do que aquele manifestado pelos demais investidores. }\end{array}$ \\
\hline Macedo Jr. (2003) & $\begin{array}{l}\text { O pesquisador utilizou de uma estratégia metodológica experimental para testar se o efeito } \\
\text { disposição entre os tomadores de decisão no ambiente simulado. Os resultados mostraram que } \\
\text { houve relutância expressiva e significante na venda de ações com prejuízos, típica do efeito } \\
\text { disposição. }\end{array}$ \\
\hline Karsten (2005) & $\begin{array}{l}\text { Valendo-se de uma amostra brasileira de dados e ainda relacionando a avaliação do efeito } \\
\text { disposição a variáveis diversas como volume de transaçãa, balanceamento de carteira e outras, o } \\
\text { pesquisador obteve resultados controversos em relação à manifestação do efeito disposição. }\end{array}$ \\
\hline Mineto (2005) & $\begin{array}{l}\text { Com metodologia semelhante àquela utilizada por Macedo Jr (2003), o pesquisador verificou } \\
\text { a influência dos pontos de referência sobre a venda de ações. Os resultados mostraram forte } \\
\text { manifestação do efeito disposição tanto entre homens como mulheres. }\end{array}$ \\
\hline Pereira (2005) & $\begin{array}{l}\text { Avaliação das operações realizadas com três fundos de investimentos brasileiros. Os resultados das } \\
\text { avaliações feitas pelo pesquisador mostram que as evidências da presença de efeito disposição são } \\
\text { inconclusivas, visto que houve indícios desse viés decisório apenas em um dos três fundos avaliados. }\end{array}$ \\
\hline Arruda (2006) & $\begin{array}{l}\text { A partir de estratégia metodológica experimental, semelhante àquelas utilizadas por Macedo Jr } \\
\text { (2003) e Mineto (2005), com objetivo de avaliar o comportamento decisório dos participantes, foi } \\
\text { constatado o efeito disposição na venda de ações tanto por homens quanto por mulheres. }\end{array}$ \\
\hline Brown et al. (2006) & $\begin{array}{l}\text { Em estudo com ações negociadas em oferta inicial (IPO), os pesquisadores buscaram identificar } \\
\text { fatores que poderiam reduzir ou mitigar o efeito disposição. Maiores experiência, nível de } \\
\text { sofisticação investidor e tempo médio das carteiras foram relevantes para reduzir o efeito disposição. }\end{array}$ \\
\hline Frazzini (2006) & $\begin{array}{l}\text { O pesquisador relacionou o efeito disposição com a divulgação de notícias sobre as companhias } \\
\text { e percebeu que há uma resposta lenta dos preços tanto para notícias positivas quanto negativas, } \\
\text { gerada pelo efeito disposição que pode fazer o ciclo de ganhos e perdas perdurar previsivelmente } \\
\text { por mais tempo. }\end{array}$ \\
\hline Barber et al. (2007) & $\begin{array}{c}\text { Por meio de método de investigação do efeito disposição mais abrangente em relação ao estudo } \\
\text { de Odean (1998), por exemplo, os pesquisadores identificaram que investidores de diversas classes } \\
\text { comentem o efeito disposição, com exceção de investidores estrangeiros e gestores de fundos } \\
\text { mútuos. }\end{array}$ \\
\hline
\end{tabular}




\begin{tabular}{|c|c|}
\hline Tizziani (2008) & $\begin{array}{c}\text { A partir da avaliação do volume de transações realizadas por fundos de investimento brasileiros, o } \\
\text { autor identificou efeito disposição em relação ao número de transação. No entanto, o mesmo não } \\
\text { ocorreu em relação ao volume monetário das operações, sendo inconclusiva a presença do viés. }\end{array}$ \\
\hline Barberis e Xiong (2009) & $\begin{array}{c}\text { Os pesquisadores realizaram simulações de investimento com base nos fundamentos da teoria } \\
\text { do prospecto a fim de verificar a incidência do efeito disposição nas decisões dos investidores. } \\
\text { Conforme a teoria subjacente, o modelo anual de ganhos e perdas realizados sustentou a } \\
\text { recorrência do efeito disposição. }\end{array}$ \\
\hline
\end{tabular}

O Quadro 1 mostra que houve uma evolução nos estudos sobre o efeito disposição, especialmente a partir dos estudos de Odean (1998), que se valeu de uma larga amostra de dados e consagrou o método de teste de comparação de proporções, que viria a ser replicado por alguns dos estudiosos ao longo do período. Os estudos nacionais citados foram dos poucos que se valeram de estratégia experimental, mostrando ser essa um método pouco utilizado para estudo deste fenômeno até 2009.

O levantamento feito por Lucchesi (2010) pode ser considerado relevante, especialmente para pesquisadores que estejam em busca de evidências na literatura sobre o efeito disposição e desejam identificar referências sobre o assunto. É preciso, contudo, consolidar os achados desses e dos estudos realizados a partir de 2010 sobre o tema. Por isso, o presente estudo verifica a produção científica sobre o efeito disposição a partir de 2010, por meio dos procedimentos metodológicos expostos a seguir, relacionando-os com os trabalhos realizados por Lucchesi (2010), a fim de consolidar as evidências dos estudos em questão.

\section{ESTUDOS EMPÍRICOS}

O presente estudo, com vistas a atingir o objetivo proposto, se sustenta em uma metodologia analítica de estudos empíricos sobre o tema efeito disposição ao longo do período de 2010 a 2015. Esse período foi selecionado intencionalmente, com o objetivo de verificar que eventuais mudanças ou achados relevantes foi identificados neste intervalo temporal que contribuem com os achados apresentados pelos estudos compilados por Lucchesi (2010), que discutiu os trabalhos produzidos desde Shefrin e Statman (1985) até 2009.

A base para o desenvolvimento desse estudo se dá a partir de um estudo bibliográfico, considerado por Martins e Theóphilo (2009) como uma estratégia que busca explicar e discutir um assunto, tema ou problema com base em referências publicadas em diversos meios. Neste caso, a fonte de pesquisa foram os artigos publicados nos diversos periódicos de pesquisa nacional e internacional indexados ao ProQuest Databases e o Google Scholar, que indexam em suas bases os principais periódicos acadêmicos.

O critério de seleção se deu pelo período da publicação, a presença do termo "disposition effect" (efeito disposição) no título e o referido trabalho ter sido citado ao menos uma vez, de modo a expressar alguma relevância no meio acadêmico. Não foram considerados trabalhos que eventualmente atendessem aos critérios de filtragem da pesquisa, mas que não se voltassem para o efeito disposição no âmbito das finanças ou mercado financeiro. Com base nesses critérios, foi possível identificar trinta e seis artigos.

A partir dessa seleção, foi realizada análise dos critérios metodológicos, do país no qual os estudos foram realizados e os achados que cada trabalho apresenta, além de suas fontes de dados empíricos. A partir daí foram construídas redes científicas, construídas com apoio do aplicativo Ucinet, para melhor expor o relacionamento entre os estudos, além de quadros sintéticos que compilam os achados. A despeito das diferenças de objetivos e conteúdos, D'Souza e Lima (2014) se valeram de metodologia semelhante, com vistas a analisar a produção científica a respeito dos estudos sobre os traços de personalidade conhecidos como Dark Triad. 


\section{REDES CIENTÍFICAS}

Do compêndio apresentado por Lucchesi (2010), nota-se que a maior parte dos estudos centra-se no estudo das bases de dados de corretoras e fundos, que apresentam as transações realizadas pelos investidores ao longo de determinado intervalo de tempo. Essa fonte de dados ainda prevalece nos estudos realizados desde 2010, conforme expressa a rede científica apresentada na Figura 1. De todo modo, é relevante o número de trabalhos que se vale de estratégias experimentais.

O acesso que alguns pesquisadores têm a base de dados de corretoras permite aos mesmos realizar estudos a partir das operações realizadas pelos investidores. Estratégias como essas foram adotadas pela maioria dos pesquisadores no período 2010 a 2014, conforme pode ser verificado na Figura 1. Em virtude de, muitas vezes, esses dados não permitirem leituras mais objetivas das evidências, há pesquisadores que optam por realizar seus estudos por meio de estratégias experimentais. Em comparação com o que é apresentado por Lucchesi (2010), há um crescimento do uso de estratégias experimentais nos estudos sobre o efeito disposição nos últimos anos. Enquanto o pesquisador identificou quatro estudos utilizando experimento, representando pouco mais de $17 \%$ dos trabalhos, neste levantamento foram identificadas onze pesquisas com esse tipo de estratégia, o que representa mais de $30 \%$ do total, conforme pode ser constatado na Figura 1.

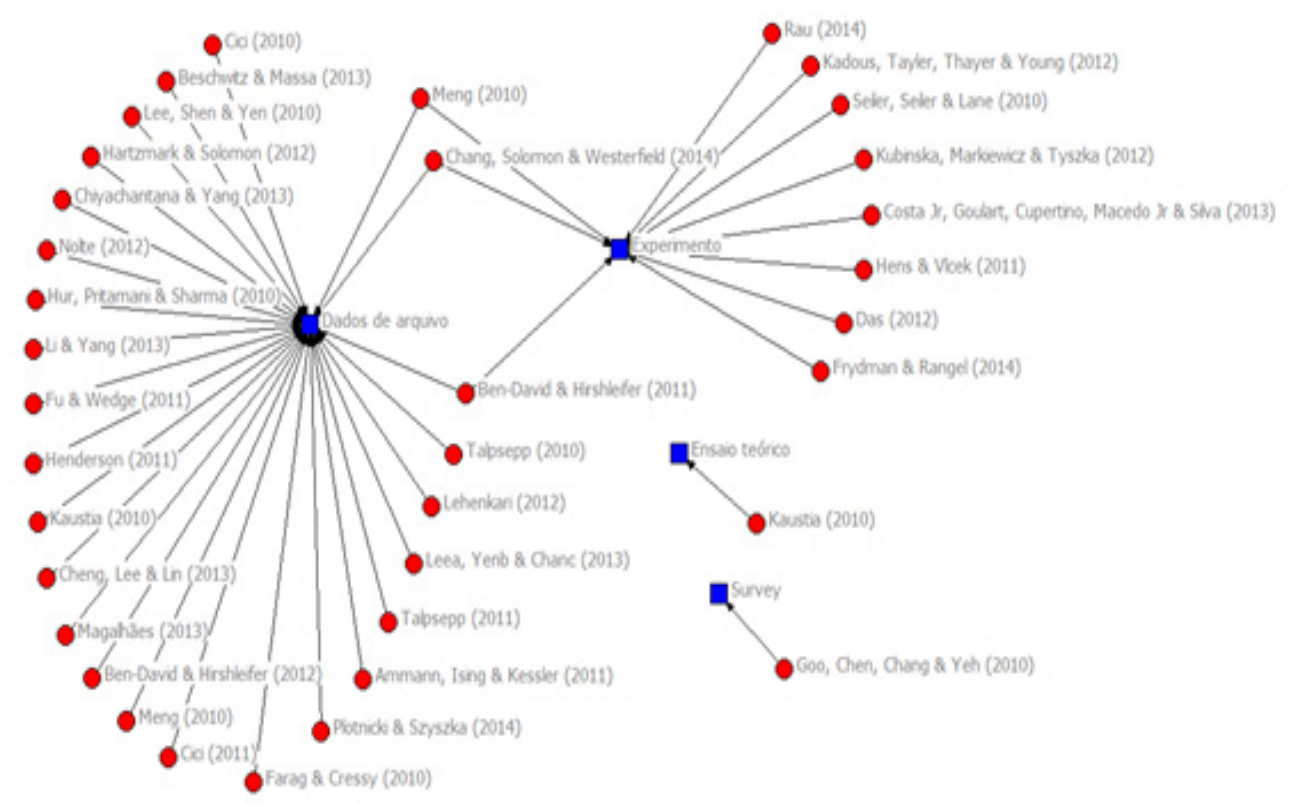

Figura 1: Rede científica das estratégias metodológicas dos estudos sobre o efeito disposição

Há ainda pesquisadores que combinam as duas estratégias, valendo-se tanto de estudos de bases de dados de corretoras ou de operações realizadas por fundos de investimentos, como também de experimentos, conforme pode ainda ser constatado na Figura 1. Com isso, é possível realizar mais testes com os dados e realizar a validação dos achados por meio de cruzamentos dos resultados.

Interessante notar que a prática de coleta de dados por meio de questionários não é comum, tendo sido realizado apenas um trabalho com uso da estratégia do levantamento (survey). A prática de experimentos ou coleta de dados históricos de operações reais se mostram mais comuns em estudos sobre o efeito disposição. Essa tendência pode se dever ao fato de os pesquisadores perceberem que 
capturar dados oriundos de ambientes reais ou que simulem a realidade permitirá capturar mais fielmente o comportamento dos agentes decisórios.

Os procedimentos metodológicos para o tratamento dos dados se distribuem desde a utilização de modelos de regressão, regressão logística, diferenças de probabilidades, além da diferença de médias e proporções, que foi originalmente utilizada por Shefrin e Statman (1985). A Figura 2 a mostra essa distribuição.

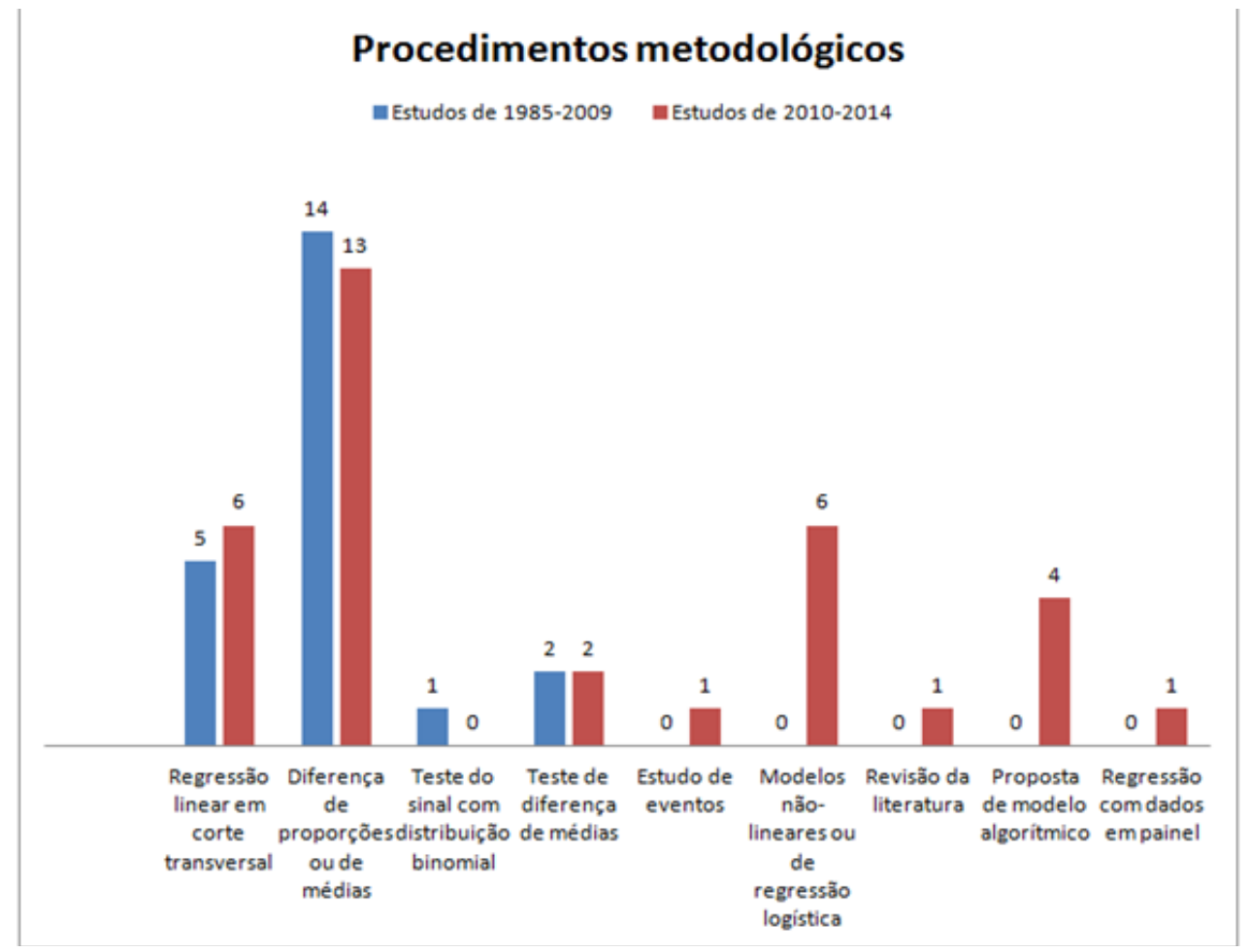

Figura 2: Metodologia dos trabalhos nos períodos 1985-2009 e 2010-2015

É evidente, pela Figura 2, que houve uma mudança em relação aos modelos estatísticos utilizados como parte do método de investigação dos trabalhos. Se no período até 2009 prevaleciam técnicas originais ou mais consagradas, como o teste de diferença de proporções, desde 2010 começam a haver uma busca por outros modelos, aliados a mudanças nas fontes de dados, que passam a se valer mais de estratégias experimentais ou de simulações.

O teste de diferença de proporções foi originalmente utilizado por Shefrin e Statman (1985) em sua pesquisa seminal sobre o efeito disposição. A partir do trabalho de Odean (1998), que prima pela larga amostra de dados utilizados, esse método estatístico passou a ser mais amplamente utilizado pelos pesquisadores, sendo o método mais utilizado, seja de maneira isolada ou combinado com outro teste, como exposto na Figura 2.

Apesar do amplo uso dos testes de diferenças de proporções e de médias, outros métodos foram propostos por pesquisadores que buscavam compreender o comportamento dos investidores em relação à prática do efeito disposição. Desse modo, a partir de 2010 os pesquisadores não apenas se valeram se outras técnicas estatísticas (Ben-David \& Hirshleifer, 2011; 2012; Hartzmark \& Solomon, 2012; Hens \& Vlcerk, 2011; Kaustia, 2010; Lehenkari, 2012; Nolte, 2012; Plotnicki \& Szyszka, 2014), como chegaram até mesmo a propor outros modelos analíticos (Henderson, 2012; Li \& Yang, 2013; Meng, 2010). 
Todo esse esforço em busca de compreender o efeito disposição no âmbito das decisões individuais dos investidores, como de gestores de fundos mútuos e até mesmo no mercado futuro e de imóveis.

A constatação a que se chega ao analisar os achados de todos esses estudos é que o efeito disposição é um evento recorrente no contexto decisório dos investidores, mas que sua compreensão não é tão simples e objetiva quanto o seu conceito. $O$ Quadro 2 a seguir apresenta a síntese dos achados dos estudos realizados entre 2010 e 2014.

Quadro 2: Síntese dos achados dos trabalhos sobre o efeito disposição no período 2010-2014.

\begin{tabular}{|c|c|}
\hline Trabalho & Síntese dos achados \\
\hline Talpsepp (2010) & $\begin{array}{c}\text { Identificada diferença de gênero em relação ao efeito disposição. Pessoas mais velhas e mulheres } \\
\text { apresentam retorno melhor em seus portfólios. }\end{array}$ \\
\hline Goo, Chen, Chang e Yeh (2010) & $\begin{array}{l}\text { Diferença de comportamento em mercados Bull e Bear. Apenas } 26 \% \text { dos investidores obtêm } \\
\text { pequenos ganhos em mercado Bull; maior nível de educação reduz o efeito disposição; o status de } \\
\text { ganhos e perdas influencia o efeito disposição; grupos perdedores são mais fortemente afetados } \\
\text { pelo efeito disposição; a evitação ao arrependimento, busca por maximização dos lucros e orgulho } \\
\text { são fatores que explicam a exposição ao efeito disposição. }\end{array}$ \\
\hline Farag e Cressy (2010) & $\begin{array}{l}\text { O efeito disposição foi identificado para venda de vencedoras e compra de perdedoras, mas fatores } \\
\text { específicos a cada companhia explicam mais do comportamento dos preços do que o efeito } \\
\text { disposição. }\end{array}$ \\
\hline Hur, Pritamani e Sharma (2010) & Diferentes estratégias utilizando o indicador momentum podem reduzir o efeito disposição. \\
\hline Meng (2010) & $\begin{array}{l}\text { O ponto de referência adotado pelos investidores pode influenciar o efeito disposição. É apresentada } \\
\text { proposta de modelo que considera novas variáveis, àquelas mais utilizadas. }\end{array}$ \\
\hline Seiler, Seiler e Lane (2010) & $\begin{array}{l}\text { Identificação dos vieses oriundos da contabilidade mental para negociação de imóveis por parte de } \\
\text { investidores, a partir de pontos de referência falsos, apontando para tendência ao efeito disposição. }\end{array}$ \\
\hline Kaustia (2010) & $\begin{array}{l}\text { A teoria do prospecto não é suficiente para explicar o efeito disposição. Dados de operações } \\
\text { mostram que a tendência a vender ações tem uma alta forte perto do retorno zero e segue constante } \\
\text { ao longo de um grande intervalo de perdas, voltando a aumentar em um grande intervalo de ganhos } \\
\text { (diferente da proposição da teoria do prospecto). }\end{array}$ \\
\hline Cici (2010) & $\begin{array}{l}\text { Em média, fundos mútuos preferem realizar perdas de capital mais a ganhos. Outra porção da } \\
\text { amostra, composta de fundos mútuos, exibe o efeito disposição, quando esses fundos não são } \\
\text { geridos por gestores com experiência em gestão de portfólios. }\end{array}$ \\
\hline Meng (2010) & $\begin{array}{c}\text { O efeito disposição se manifesta por meio da aversão a perda, quando o ponto de referência é } \\
\text { definido pela riqueza esperada e não quando o ponto de referência é definido pela riqueza inicial. } \\
\text { Quando os investidores não atualizam os seus pontos de referência, o efeito disposição se manifesta } \\
\text { mais frequentemente. }\end{array}$ \\
\hline Lee, Shen e Yen (2010) & $\begin{array}{l}\text { A tendência a apresentar o efeito disposição nos fundos mútuos depende de fatores associados ao } \\
\text { contexto de mercado. }\end{array}$ \\
\hline Fu e Wedge (2011) & $\begin{array}{l}\text { Fundos mútuos com proprietários gestores exigem menor grau de efeito disposição do que os não } \\
\text { têm proprietários como gestores. }\end{array}$ \\
\hline Cici (2012) & $\begin{array}{l}\text { Fundos mútuos geridos por gestores experientes exibem menor efeito disposição, afetando o } \\
\text { desempenho dos fundos. }\end{array}$ \\
\hline Talpsepp (2011) & $\begin{array}{c}\text { Investidores estrangeiros, quando comparados a investidores locais, apresentam tendência ao } \\
\text { efeito disposição "reverso" (vender perdedoras com mais frequência). Experiência e sofisticação do } \\
\text { investidor parecem reduzir o efeito disposição. }\end{array}$ \\
\hline Hens e Vlcek (2011) & $\begin{array}{l}\text { O efeito disposição não pode ser plenamente explicado pela teoria do prospecto, uma vez que não } \\
\text { há uma referência de investimento anterior para explicar o efeito disposição, como sugere a teoria } \\
\text { do prospecto. }\end{array}$ \\
\hline Ammann, Ising e Kessler (2011) & $\begin{array}{l}\text { Forte evidência de efeito disposição em fundos mútuos, com forte correlação entre efeito disposição } \\
\text { em fundos mútuos e mudanças no ambiente macroeconômico. Os gestores menos propensos ao } \\
\text { efeito disposição investem grandes volumes em grandes companhias. }\end{array}$ \\
\hline Ben-David e Hirshleifer (2011) & $\begin{array}{l}\text { O efeito disposição não é o fator preponderante no processo de decisão de negociação. } \mathrm{O} \\
\text { movimento especulativo pode explicar parte dos aspectos motivacionais às decisões, que levam ao } \\
\text { efeito disposição reverso. }\end{array}$ \\
\hline Henderson (2011) & $\begin{array}{l}\text { O investidor desiste do ativo e realiza prejuízo, quando o ativo exibe índice de Sharpe } \\
\text { suficientemente baixo. }\end{array}$ \\
\hline Ben-David e Hirshleifer (2012) & $\begin{array}{l}\text { Não há uma explicação simples e direta do efeito disposição, sendo este explicado pelas crenças do } \\
\text { investidor e a mudança de suas preferências. }\end{array}$ \\
\hline Lehenkari (2012) & $\begin{array}{l}\text { O efeito disposição se manifesta quando o investidor é pessoalmente responsável pelas decisões em } \\
\text { relação ao portfólio, sendo potencialmente explicado pela "escalada do comprometimento". }\end{array}$ \\
\hline Das (2012) & $\begin{array}{l}\text { Negociação mais frequente não reduz o efeito disposição; disciplina nas negociações de ações, que } \\
\text { incluem tardar a venda de ações vencedoras, reduzem as perdas atribuídas ao efeito disposição. }\end{array}$ \\
\hline Kadous, Tayler, Thayer e Young (2012) & $\begin{array}{l}\text { Influencia comportamental afeta o efeito disposição. Investidores com baixo "amor-próprio" mantêm } \\
\text { investimentos perdedores por mais tempo (maior efeito disposição). }\end{array}$ \\
\hline Kubińska, Markiewicz e Tyszka (2012) & $\begin{array}{l}\text { Investidores que utilizam o indicador momentum como estratégia estão menos sujeitos ao efeito } \\
\text { disposição do que os demais investidores. }\end{array}$ \\
\hline
\end{tabular}




\begin{tabular}{|c|c|}
\hline Hartzmark e Solomon (2012) & $\begin{array}{c}\text { Má formação de preços e expectativa de reversão à média levam os investidores a exibirem o efeito } \\
\text { disposição. }\end{array}$ \\
\hline Nolte (2012) & $\begin{array}{l}\text { A presença do efeito disposição é influenciada por diversos aspectos relacionados à estratégia do } \\
\text { investidor. Para pequenos lucros ou perdas, o efeito disposição é invertido. O efeito disposição } \\
\text { invertido aparece para investidores pacientes e cautelosos que se valem de ordens programadas } \\
\text { (stop loss e gain). O efeito disposição normal aparece em investidores impacientes que realizam } \\
\text { operações ativas no mercado. Grandes investidores manifestam menos efeito disposição do que os } \\
\text { pequenos investidores. }\end{array}$ \\
\hline Cheng, Lee e Lin (2013) & $\begin{array}{l}\text { Mulheres e homens maduros apresentam mais efeito disposição e o mercado "Bear" apresenta maior } \\
\text { efeito disposição. }\end{array}$ \\
\hline Magalhães (2013) & $\begin{array}{l}\text { Existência de efeito disposição mais em mercados "Bull" do que "Bear" em fundos de ações } \\
\text { portugueses. }\end{array}$ \\
\hline Leea, Yenb e Chanc (2013) & $\begin{array}{l}\text { Os investidores de fundos mútuos resgatam suas cotas com mais frequência em mercados "Bear" do } \\
\text { que em mercados "Bull". O efeito disposição aparece de acordo com o comportamento do mercado, } \\
\text { mas não de forma homogênea. }\end{array}$ \\
\hline $\begin{array}{l}\text { Costa Jr, Goulart, Cupertino, Macedo Jr e Silva } \\
\qquad \text { (2013) }\end{array}$ & $\begin{array}{l}\text { Investidores mais experientes exibem menos efeito disposição quando comparados aos investidores } \\
\text { inexperientes. }\end{array}$ \\
\hline Li e Yang (2013) & $\begin{array}{l}\text { Proposta de modelo matemático com uso de métricas referentes a preço momentum, retorno, } \\
\text { volatilidade, modelo busca entender critérios associados a preço de ativos e efeito disposição. }\end{array}$ \\
\hline Chiyachantana e Yang (2013) & $\begin{array}{l}\text { Importância do ponto de referência mostra que a inabilidade dos investidores em definir preços de } \\
\text { referência gera efeito disposição. Expectativas negativas em relação a resultados futuros aumentam a } \\
\text { chance de realização de perdas. }\end{array}$ \\
\hline Beschwitz e Massa (2013) & $\begin{array}{l}\text { Analise do efeito disposição em posições vendidas (short). Os vendedores apresentam tendência } \\
\text { a cometer vieses comportamentais, devido a preconceitos em relação à formação dos preços do } \\
\text { mercado (não fazem simplesmente arbitragem de preços). }\end{array}$ \\
\hline Rau (2014) & $\begin{array}{l}\text { O estudo envolvendo diferença de gênero mostra que as mulheres são mais avessas a risco e menos } \\
\text { propensas a realizar perdas do que os homens. }\end{array}$ \\
\hline Plotnicki e Szyszka (2014) & $\begin{array}{l}\text { Existência de efeito disposição por parte dos gestores na decisão de realizar IPO (abertura de capital). } \\
\text { Eles entram mais rápido quando o mercado está em alta e obtém retornos menores. }\end{array}$ \\
\hline Chang, Solomon e Westerfield (2014) & $\begin{array}{l}\text { Existência de efeito disposição apenas para ativos com gestão não delegada (ações individuais), não } \\
\text { para fundos (ativos delegados). }\end{array}$ \\
\hline Frydman e Rangel (2014) & $\begin{array}{c}\text { As pessoas tendem a apresentar maior efeito disposição quando referências de preços de compras } \\
\text { são apresentadas a elas. Seria possível reduzir o efeito disposição se a informação (saliência) sobre os } \\
\text { preços de compra fossem omitidas nos relatórios financeiros. }\end{array}$ \\
\hline
\end{tabular}

A síntese apresentada no Quadro 2 deixa claro que o efeito disposição tem muitas faces e suas explicações estão longe de serem conclusivas. Ao se comparar o conteúdo do Quadro 2 com aquele exposto no Quadro 1, nota-se que há uma busca por verificar se o efeito disposição se manifesta em outros contextos, por meio de novos métodos, a fim de identificar evidências que possam melhor explicá-lo, ainda que lacunas possam ser identificadas.

Lucchesi (2010) já apontava para tal fato, uma vez que em seu estudo destaca a controvérsia em relação ao efeito disposição. Em seus próprios achados o pesquisador conseguiu, por meio de estudos envolvendo a aplicação da regressão logística, identificar a presença do efeito disposição em fundos de investimentos brasileiros, mas não conseguiu sustentar a explicação da reversão à média tão comumente difundida.

Complementando os resultados do Quadro 2, considerando estudos brasileiros, mais recentemente é identificado estudo recente de Lucchesi, no qual o pesquisador reforça os achados de sua pesquisa anterior e a controvérsia sobre as causas do efeito disposição (Lucchesi, Yoshinaga \& Castro Jr, 2015). Ainda que reconheçam que os fundamentos da teoria do prospecto expliquem parcialmente o comportamento dos agentes, não há validação da hipótese da reversão à média.

Resultados como esses têm feito com que as pesquisas mais recentes sobre o efeito disposição busque identificar variáveis ou fatores que possam explicar sua existência. Se no período anterior as pesquisas caminhavam para validar o efeito disposição, os trabalhos mais recentes não questionam sua existência, mas querem identificar suas causas e explicações. A comparação dos trabalhos do período anterior a 2010 e aqueles realizados posteriormente sinalizam para essa mudança.

Se, por um lado, as pesquisas mostram que o efeito disposição é um viés decisório que tende a afetar os tomadores de decisões em diversos contextos que envolvem ganhos e perdas, por outro, não estão 
plenamente claros os motivos que fazem os agentes sucumbirem a esse viés. Ou seja, sabe-se que efeito disposição leva o investidor a realizar lucros muito antes das perdas, mas as causas e circunstâncias nas quais ele tem atraído atenção dos pesquisadores e têm sido o foco dos estudos mais recentes.

Os resultados apresentados no presente estudo reforçam a relevância desse viés decisório no âmbito das pesquisas sobre comportamento decisório dos agentes na linha das finanças comportamentais. A busca por utilizar amostras, métodos e técnicas estatísticas diferenciadas mostra que os estudos mais recentes estão interessados em encontrar elementos novos que contribuam com potenciais explicações para o efeito disposição. 


\section{CONSIDERAÇÕES FINAIS}

O efeito disposição é um fenômeno que se configura pela tendência que as pessoas têm de realizar ganhos mais cedo do que as perdas. Esse comportamento não se sustenta nos fundamentos considerados racionais da teoria econômica, comprometendo a maximização de ganhos por parte dos investidores. Desde os estudos seminais oriundos da teoria do prospecto, várias pesquisas foram realizadas sobre o efeito disposição e suas variantes, seja no mercado financeiro, no mercado imobiliário, nas decisões de investidores individuais ou por gestores de fundos mútuos.

Frente à controvérsia em relação ao entendimento e validação do efeito disposição, o presente estudo buscou analisar a produção científica realizada sobre o tema desde 2010. Por meio de um levantamento bibliográfico, uso de redes científicas e metodologia empírico-analítica foi possível identificar que se trata de um tema muito recorrente na literatura recente sobre decisões de investimento, cujos achados estão longe de serem conclusivos.

O efeito disposição apresenta-se como um assunto com múltiplas facetas e oportunidades de estudos, uma vez que as explicações obtidas pelas pesquisas científicas realizados no período analisado apontam para diferentes horizontes, mostrando que ainda há muito que se investigar sobre o comportamento do investidor. Não há de se assumir uma visão linear sobre a tendência a realização de ganhos e perdas por parte dos indivíduos.

Com base no levantamento feito no presente estudo, fica clara a evolução dos instrumentos metodológicos e das técnicas estatísticas empregadas nos estudos desde 2010. O evidente interesse nos pesquisadores pelo efeito disposição se expressa no maior número de trabalhos identificados no período. A aplicação dos fundamentos desse viés cognitivo em outros contextos de investimento também pode ser considerada uma evolução, ainda que residam contradições quanto aos fatores que expliquem a tendência da venda de ativos com ganhos e perdas.

O que isso pode representar para o futuro deve ser motivo de mais pesquisas, de modo a responder questões que derivam dos achados apresentados pelos trabalhos investigados aqui. Resguardadas as naturais limitações que um estudo desta natureza traz consigo, este há de um ser um estudo que poderá servir como ponto de partida para pesquisadores que queiram dar continuidade às investigações sobre o efeito disposição, deixando muito mais questionamentos do que respostas, como é típico da própria ciência. 


\section{REFERÊNCIAS}

Ammann, M., Ising, A., \& Kessler, S. (2011). Disposition effect and mutual fund performance. Applied Financial Economics, 22(1): 1-19.

Barber, B. M., \& Odean, T. (2000). Trading is hazardous to your wealth: the common stock investment performance of individual investors. The Journal of Finance, 55(2): 773-806.

Barber, B. M., \& Odean, T. (2001). Boys will be boys: gender, overconfidence, and common stock investment. The Quarterly Journal of Economics, 116(1): 261-292.

Ben-David, I., \& Hirshleifer, D. (2011). Are investors really reluctant to realize their losses? Trading responses to past returns and the disposition effect. Review of Financial Studies, 25(8): 2485-2532.

Ben-David, I., \& Hirshleifer, D. (2012). Beyond the disposition effect: do investors really like realizing gains more than losses. Review of Financial Studies, 25(8): 2485-2532.

Beschwitz, B., Massa, M. (2013). Biased shorts: stock market implications of short sellers' disposition effect. Available at SSRN 2348583.

Chang, T., Solomon, D. H., \& Westerfield, M. M. Looking for someone to blame: delegation, cognitive dissonance, and the disposition effect. <http://fisher.osu.edu/supplements/10/12706/CSW-20130520. pdf >. 2014. Acesso em 14/02/2015.

Cheng, T. Y., Lee, C. I., \& Lin, C. H. (2013). An examination of the relationship between the disposition effect and gender, age, the traded security, and bull-bear market conditions. Journal of Empirical Finance, (21): 195-213.

Chiyachantana, C. N., Yang, Z. (2013). Reference point adaptation and disposition effect. < http://ink. library.smu.edu.sg/cgi/viewcontent.cgi?article $=4784 \&$ context=lkcsb_research $>$. Acesso em 14/02/2015.

Cici, G. (2010). The relation of the disposition effect to mutual fund trades and performance. Available at SSRN 645841.

Cici, G. (2012). The prevalence of the disposition effect in mutual funds'trades. Journal of Financial and Quantitative Analysis, 47(04): 795-820.

Costa Junior, N., Goulart, M., Cupertino, C., Macedo, J., \& Silva, S. (2013). The disposition effect and investor experience. Journal of Banking \& Finance, 37(5): 1669-1675.

Costa Junior, N., Mineto, C., \& Silva, S. (2008). Disposition effect and gender. Applied Economics Letters, 15(6): 411-416.

Das, A. (2012). Estimating the loss from the disposition effect: a simulation study. Journal of Behavioral Finance, 13(1): 1-10.

Deweaver, M. A., \& Shannon, R. (2010). Waning vigilance and the disposition effect: evidence from Thailand on individual investor decision making. The Journal of Socio-Economics, 39(1): 18-23.

D'souza, M.F.; \& Lima, G.A.S.F. (2015). Taxonomia do Dark Triad: revelações da rede científica no meio organizacional. In: Anais $14^{\circ}$ Congresso USP de Controladoria e Contabilidade. Recuperado em 10 de abril de 2015, de: http://www.congressousp. fipecafi.org/anais_congresso.aspx.

Farag, H., \& Cressy, R. (2010). Do unobservable factors explain the disposition effect in emerging stock markets? Applied Financial Economics, 20(15): 1173-1183. 
Ferreira, V. R. M. (2008). Psicologia Econômica: estudo do comportamento econômico e da tomada de decisão. Elsevier, Rio de Janeiro.

Frydman, C., \& Rangel, A. (2014). Debiasing the disposition effect by reducing the saliency of information about a stock's purchase price. Journal of Economic Behavior \& Organization, (107): 541-552.

Fu, R., \& Wedge, L. (2011). Managerial ownership and the disposition effect. Journal of Banking \& Finance, 35(9): 2407-2417.

Goo, Y. J., Chen, D. H., Chang, S. H. S., \& Yeh, C. F. (2010). A study of the disposition effect for individual investors in the Taiwan stock market. Emerging Markets Finance and Trade, 46(1): 108-119.

Grinblatt, M., \& Han, B. (2004). Prospect theory, mental accounting, and momentum. Journal of Financial Economics, 78(2): 311-339.

Hartzmark, S. M., \& Solomon, D. H. (2012). Efficiency and the disposition effect in NFL prediction markets. The Quarterly Journal of Finance, 2(03): 17-58.

Henderson, V. (2012). Prospect theory, liquidation, and the disposition effect. Management Science, 58(2): 445-460.

Hens, T., \& Vlcek, M. (2011). Does prospect theory explain the disposition effect? Journal of Behavioral Finance, 12(3): 141-157.

Hur, J., Pritamani, M., \& Sharma, V. (2010). Momentum and the disposition effect: the role of individual investors. Financial Management, 39(3): 1155-1176.

Kadous, K., Tayler, W. B., Thayer, J. M., \& Young, D. (2014). Individual characteristics and the disposition effect: the opposing effects of confidence and self-regard. Journal of Behavioral Finance, 15(3): 235-250.

Kahneman, D. (2012). Rápido e devagar: duas formas de pensar. Objetiva, São Paulo.

Kahneman, D., \& Tversky, A. (1979). Prospect theory: an analysis of decisions under risk. Econometrica, (47): 313-327.

Kahneman, D., Knetsch, J., \& Thaler, R. (1990). Experimental tests of the endowment effect and the the Coase theorem. The Journal of Political Economy, 98(6): 1325-1348.

Kaustia, M. (2010). Prospect theory and the disposition effect. Cambrige Press, Cambridge.

Kubińska, E., Markiewicz, Ł., \& Tyszka, T. (2012). Disposition effect among contrarian and momentum investors. Journal of Behavioral Finance, 13(3): 214-225.

Lee, J. S., Shen, C. H., \& Yen, P. H. (2010). The study of the fund investors' disposition effect vis-à-vis herding redemption and non-herding redemption: evidence from quantile regression least square dummy variable estimator. Journal of Management and System, 17(1): 2-26.

Lee, J. S., Yen, P. H., \& Chan, K. C. (2013). Market states and disposition effect: evidence from Taiwan mutual fund investors. Applied Economics, 45(10): 1331-1342.

Lehenkari, M. (2012). In search of the underlying mechanism of the disposition effect. Journal of Behavioral Decision Making, 25(2): 196-209.

Li, Y., \& Yang, L. (2013). Prospect theory, the disposition effect, and asset prices. Journal of Financial Economics, 107(3): 715-739. 
Lucchesi, E. P. (2010). O efeito disposição e suas motivações comportamentais: um estudo com base na atuação de gestores de fundos de investimento em ações. Tese de Doutorado em Administração, Faculdade de Economia, Administração e Contabilidade da Universidade de São Paulo.

Lucchesi, Eduardo Pozzi, Yoshinaga, Claudia Emiko, Castro Jr, Francisco Henrique Figueiredo. (2015). Efeito disposição entre gestores brasileiros de fundos de ações. RAE-Revista de Administração de Empresas, 55(1): 26-37.

Magalhães, C. I. S. (2013). O efeito disposição nos fundos de investimento de acçoes portugueses. Dissertação de Mestrado em Finanças, Faculdade de Economia da Universidade do Porto.

Markowitz, H. (1952). Portfolio selection. The Journal of Finance, 7(1): 77-91.

Martins, G. A., \& Theóphilo, C. R. (2009). Metodologia da investigação científica para ciências sociais aplicadas. Atlas, São Paulo.

Meng, J. (2010). The disposition effect and expectations as reference point. <http://www.wiwi.uni-bonn. de/kraehmer/Lehre/Beh_Econ/Papiere/Meng-Disposition-Effect.pdf>. Acesso em 16/02/2015.

Nolte, I. (2012). A detailed investigation of the disposition effect and individual trading behavior: a panel survival approach. The European Journal of Finance, 18(10): 885-919.

Odean, T. (1998). Are investors reluctant to realize their losses? The Journal of Finance, 53(5): 1775-1798.

Plotnicki, M., \& Szyszka, A. (2014). IPO market timing: the evidence of the disposition effect among corporate managers. Global Finance Journal, 25(1): 48-55.

Rau, H. A. (2014). The disposition effect and loss aversion: do gender differences matter? Economics Letters, 123(1): 33-36.

Samuelson, W., \& Zeckhauser, R. (1988). Status quo bias in decision making. Journal of Risk and Uncertainty, 1(1): 7-59.

Seiler, M. J., Seiler, V. L., \& Lane, M. A. (2012). Mental accounting and false reference points in real estate investment decision making. Journal of Behavioral Finance, 13(1): 17-26.

Shefrin, H., \& Statman, M. (1985). The disposition to sell winners too early and ride losers too long: theory and evidence. The Journal of Finance, 40(3): 777-790.

Talpsepp, T. Reverse disposition effect of foreign investors. Journal of Behavioral Finance, 12(4): 183-200.

Talpsepp, T. (2013). Does gender and age affect investor performance and the disposition effect? Research in Economics and Business: Central and Eastern Europe, 2(1): 1-18.

Talpsepp, T., Vlcek, M., \& Wang, M. (2014). Speculating in gains, waiting in losses: a closer look at the disposition effect. Journal of Behavioral and Experimental Finance, (2): 31-43.

Thaler, R. (1985). Mental accounting and consumer choice. Marketing Science, 4(3): 199-214.

Thaler, R. H., \& Sunstein, C. R. (2008). Nudge: improving decisions about health, wealth, and happiness. Yale University Press, New Haven. 